Article

\title{
Reference Expression Profile of Three FBN1 Transcript Isoforms and Their Association with Clinical Variability in Marfan Syndrome
}

\author{
Louise Benarroch ${ }^{1}(0)$, Mélodie Aubart ${ }^{1,2}$, Marie-Sylvie Gross ${ }^{1}$, Pauline Arnaud ${ }^{1,3}(0)$ \\ Nadine Hanna ${ }^{3}$, Guillaume Jondeau ${ }^{1,4,5,+}$ and Catherine Boileau $1,3,5, *,+$ (D) \\ 1 Laboratory for Vascular Translational Science, INSERM U1148, Centre Hospitalo-Universitaire Xavier Bichat, \\ 46 rue Henri Huchard, 75018 Paris, France; louise.benarroch@inserm.fr (L.B.); \\ melodie.aubart@inserm.fr (M.A.); marie-sylvie.gross@inserm.fr (M.-S.G.); pauline.arnaud@inserm.fr (P.A.); \\ guillaume.jondeau@inserm.fr (G.J.) \\ 2 Service de Neuropédiatrie, Hôpital Necker-Enfants-Malades, 149 rue de Sèvres, 75015 Paris, France \\ 3 Département de Génétique, Centre Hospitalo-Universitaire Xavier Bichat, 46 rue Henri 17 Huchard, \\ 75018 Paris, France; nadine.hanna@aphp.fr \\ 4 Centre de Référence pour le Syndrome de Marfan et syndromes apparentés, Service de Cardiologie, \\ Centre Hospitalo-Universitaire Xavier Bichat, 46 rue Henri Huchard, 75018 Paris, France \\ 5 UFR de Médecine, Université Paris Diderot, 16 rue Henri Huchard, 75018 Paris, France \\ * Correspondence: catherine.boileau@inserm.fr; Tel.: +33-1-40-25-75-31-21 \\ $\dagger$ These authors contributed equally.
}

Received: 7 January 2019; Accepted: 6 February 2019; Published: 11 February 2019

\begin{abstract}
Marfan syndrome (MFS) is a rare connective tissue disorder mainly due to mutations in the FBN1 gene. Great phenotypic variability is notable for age of onset, the presence and absence, and the number and the severity of the symptoms. Our team showed that $F B N 1$ gene expression level was a good surrogate endpoint for severity of some MFS clinical features. Eight alternative transcripts are referenced for the FBN1 gene. We hypothesized that MFS clinical variability could be related to specific $F B N 1$ isoforms. Isoform expression profiles were investigated in skin and adventitial fibroblasts from controls and MFS patients. The results of the study showed that, in skin and adventitial fibroblasts, only three isoforms were found: FBN1_001, FBN1_004, and FBN1_009. The main isoform was FBN1_001 and it was significantly reduced in skin and adventitial fibroblasts of MFS patients. The expressions of FBN1_004 and FBN1_009 isoforms were similar between controls and MFS patients. However, the expression of the three isoforms was correlated only in patients. Furthermore, their expression levels were associated with the presence of ectopia lentis in MFS patients. Therefore, our results highlight that the two minor alternatively spliced FBN1 isoforms play a possible role in the pathogenesis of the disease.
\end{abstract}

Keywords: Marfan syndrome; clinical variability; fibrilline-1; alternative splicing; isoforms

\section{Introduction}

Marfan syndrome (MFS; Online Mendelian Inheritance in Man database (OMIM) \#154700) is an autosomal dominant connective tissue disorder with an estimated prevalence of one in every 5000 individuals. It is a multisystemic disease that affects the ocular, skeletal, and cardiovascular systems, as well as lung, skin, and dura. In most cases, MFS is due to mutations in the FBN1 gene encoding fibrillin-1, an extra-cellular matrix protein. To date, over 3000 mutations were reported in the FBN1 database [1]. The syndrome displays great clinical variability both between and within families, with respect to the age of onset, the presence or the absence, and the number and the severity of the 
symptoms. This variability is taken into account in the last nosology for MFS with which the diagnosis can be made with or without ectopia lentis or aortic aneurysm [2]. Clinical variability cannot be explained by the few and mild genotype-phenotype correlations identified between FBN1 mutations and MFS features [3-11].

Our team pioneered the hypothesis that MFS severity could be related to the variable level of fibrillin-1 synthesized from the wild-type (WT) allele. To investigate this hypothesis, we firstly established FBN1 expression in skin fibroblasts from controls as reference values and then in MFS patients [12]. Results in controls showed a 3.9-fold variation in FBN1 messenger RNA (mRNA) synthesis level. A similar 4.4-fold variation was found in the MFS population, while the mean level of FBN1 gene expression level was half that of the control population. This study showed also that the FBN1 gene expression level was a good surrogate endpoint for severity of some MFS clinical features. Indeed, lower FBN1 gene expression levels were found to be significantly associated with ectopia lentis.

Alternative splicing is an essential biological process, and differential expression of alternative transcripts can be involved in specific biological or pathological roles. A published study on alternative splicing of the FBN1 gene showed that one FBN1 isoform's (no longer referenced in the Ensembl database) expression represented a significant proportion of total FBN1 gene expression, and that this proportion varied in a tissue- and development-specific manner [13]. Therefore, alternative splicing of the FBN1 gene could be a potential mechanism that would explain part of the clinical variability observed in MFS. To investigate this hypothesis, the expression profiles of FBN1 isoforms were investigated firstly in skin fibroblasts from control subjects to establish reference patterns. Expressions of the validated isoforms were then investigated in MFS patients, and possible correlations with clinical features were investigated.

The results of the study showed that, in skin and adventitial fibroblasts, only three isoforms were found: FBN1_001,FBN1_004, and FBN1_009. The main isoform was FBN1_001 and it was significantly reduced in skin and adventitial fibroblasts from thoracic aortic aneurysm (TAA) patients, as well as in MFS patients. The expressions of FBN1_004 and FBN1_009 isoforms were similar between controls and MFS patients, and their expression levels were associated with the presence of ectopia lentis in MFS patients. The alternative splicing mechanism, giving rise to these isoforms, remains unclear; however, these results suggest a role of this mechanism in MFS clinical variability.

\section{Materials and Methods}

\subsection{Patient and Control Samples}

MFS patients were recruited in the "Centre National Maladies Rares-Syndrome de Marfan et apparentés", the French National Reference Centre located at Bichat Hospital (Paris, France). An in-depth clinical investigation was performed, as well as imaging and slit-lamp ocular examination, to establish a systemic score and perform diagnosis of Marfan syndrome according to the revised Ghent nosology, as already reported in Reference [11]. In brief, the seven different organ systems (skin and integument, skeleton, eye, cardiovascular, neurology, and lung) were carefully examined. For the purpose of this study, adventitial fibroblasts were extracted from five patients with TAA who underwent aortic surgery (two TGFBR2 mutation carriers, one FBN1 mutation carrier, and two with aortic aneurysm of undetermined origin). For these patients, skin fibroblasts were also available and were used to investigate the relevance of FBN1 isoform expression in skin fibroblasts with respect to expression in adventitial fibroblasts. Skin fibroblasts from 42 MFS patients carrying a premature termination codon (PTC) FBN1 gene mutation were selected as a representative subset of the patient population used in Aubart et al. (2015). To develop and validate the various tests, skin fibroblasts from 15 control subjects were used. All patients gave their written informed consent for participation in this study in agreement with the requirements of French regulations (accepted by the "Comité 
de Protection des Personnes CPP Ile de France XI", 78105 St Germain en Laye, with the registration number \#11008).

\subsection{Cell Culture and RNA Purification}

Skin fibroblasts were cultivated in Dulbecco's modified Eagle medium (DMEM; Thermo Scientific, Villebon sur Yvette, France) supplemented with $4.5 \mathrm{~g} / \mathrm{L}$ glucose, $15 \%$ fetal bovine serum (FBS; PAA Laboratory, Villacoublay, France), and antibiotics (50 U/mL penicillin, streptomycin, and amphotericin B) (PAA Laboratory, Villacoublay, France), as previously described [12].

From fibroblast culture, total RNAs were extracted with the RNeasy kit ${ }^{\circledR}$ (Qiagen S.A., Courtaboeuf, France) according to the manufacturer's instructions, as previously described [14].

\subsection{Real-Time Quantitative PCR (RT-qPCR)}

Reverse transcription was performed using the miScript II RT kit (Qiagen S.A.), according to the manufacturer's recommendations. The final concentration of complementary DNA (cDNA) was $100 \mathrm{ng} / \mu \mathrm{L}$ for each sample. Real-time quantitative PCR (RT-qPCR) was performed as previously described [14]. Specific primers (Table 1) were used to quantify the gene expression of FBN1 transcripts, $S D H A$, and GAPDH. Total FBN1 gene expression was calculated by summing the expressions of the three isoforms detected by RT-qPCR.

Table 1. Primer sequences for gene expression quantification.

\begin{tabular}{|c|c|c|}
\hline Name & Forward & Reverse \\
\hline FBN1_001 & AGTCGGGCCAAGAGAAGAGGCG & TCCATCCAGGGCAACAGTAAGCAT \\
\hline FBN1_004 & TTTTACTGCTGTCTCCAGCTTTCC & ACAGCAGCATTCCGATTTGGTG \\
\hline FBN1_009 & AAACTCATGGTTTTCССССТTCT & TGATGTCTTGGCATCCTCCAC \\
\hline GAPDH & GTCGCCAGCCGAGCCACATC & CCAGGCGCCCAATACGACCA \\
\hline SDHA & AAGGGCTCCGACTGGCTGGGG & TTTCTAGCTCGACCACGGCGGC \\
\hline
\end{tabular}

\subsection{Statistical Analysis}

Statistical analysis was performed using GraphPad Prism version 7.0 software and Jump 7.0.1 software (SAS Institute Inc., Cary, NC, USA). The statistical difference between two groups was tested using an unpaired Student's $t$-test, while that between three groups was tested using one-way ANOVA or a Kruskal-Wallis test. The coefficient of determination $\left(R^{2}\right)$ was calculated by linear regression, and the statistical significance was determined with Pearson correlation. The significance was set at a $p$-value $<0.05$ for all tests.

\section{Results}

\subsection{Expression of FBN1 Isoforms in Skin Fibroblasts from Controls}

In silico analysis of $F B N 1$ isoforms showed the existence of reports for eight isoforms listed in the Ensembl database [15]. However, none of these isoforms are reported in the literature. In the Genotype-Tissue Expression (GTEx) consortium database [16], the expressions of three of the eight isoforms are reported in transformed fibroblasts and in the aorta: FBN1_001 (ENST00000316623.5, NM_000138), FBN1_004 (ENST00000559133.1), and FBN1_009 (ENST00000561429.1) (Figure 1a). The existence of all eight isoforms in control skin fibroblasts was investigated using RT-qPCR and specific primer pairs. Only isoforms FBN1_001,FBN1_004, and FBN1_009 were found. The FBN1_001 isoform was by far the major isoform found. Furthermore, in our model, FBN1_009 expression was higher than that of FBN1_004 (Figure 1b), unlike what is observed in the GTEx database. 
(a)

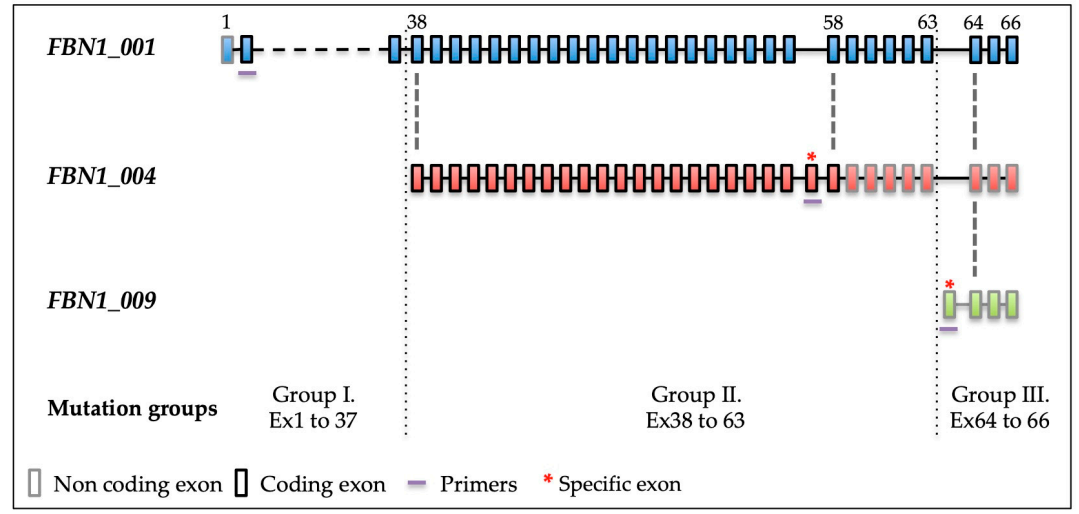

(b)

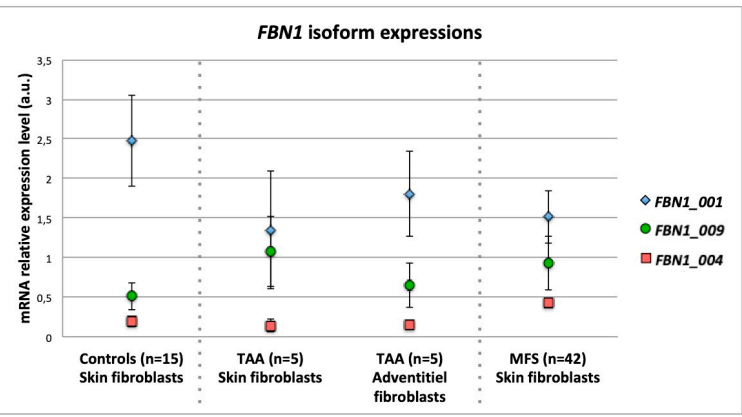

(c)
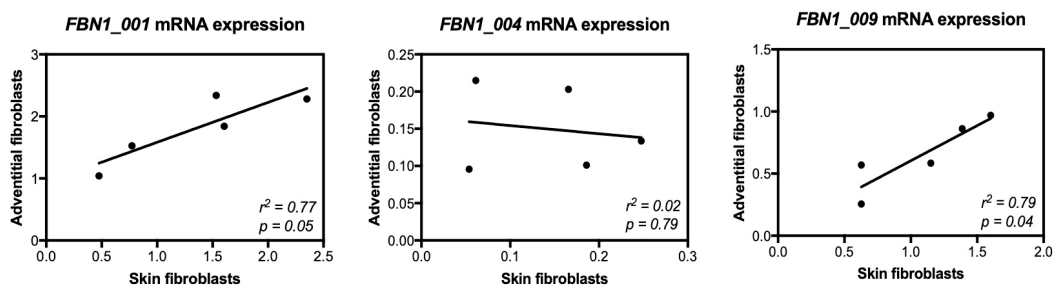

Figure 1. Description of expressed FBN1 isoforms. (a) In silico analysis of FBN1 isoforms expressed in skin fibroblasts from controls and Marfan syndrome (MFS) patients. Colored boxes represent exons, and coding (boxes with black borders) and non-coding (boxes with gray borders) regions. Exons are numbered in accordance with the referenced sequence (FBN1_001 isoform) above the boxes. Bold red asterisks indicate exons specific to each isoform. The purple lines indicate the localization of each set of primers used to quantify the expression of each isoform. MFS patients were divided into three groups depending on where the mutation was located: group I (mutations in exons 1 to 37, included), group II (exons 38 to 63, included), and group III (exons 64 to 66, included). (b) FBN1 isoform expressions in skin and adventitial fibroblasts. Quantification using real-time quantitative PCR (RT-qPCR) was performed in skin fibroblasts from 15 controls and 42 MFS patients and in adventitial fibroblasts from five patients with thoracic aortic aneurysm (TAA). (c) Correlation of expression between skin and adventitial fibroblasts. Comparison of FBN1 messenger RNA (mRNA) level for five TAA patients.

\subsection{Well-Correlated Expression of FBN1 between Skin and Aortic Adventitial Fibroblasts}

To investigate isoform expression in adventitial fibroblasts and to compare expression levels between adventitial and skin fibroblasts, we used samples from five TAA patients for whom both cell types were available. The three skin isoforms were also found in adventitial fibroblasts in agreement with GTEx data. Furthermore, skin and aortic expression levels were comparable for all three isoforms with isoform FBN1_001 as the major isoform (Figure 1b). Finally, a comparison of $F B N 1$ isoform expressions in adventitial and skin fibroblast pairs showed a positive correlation for 
FBN1_001 $\left(R^{2}=0.77 ; p=0.049\right)$ and for FBN1_009 $\left(R^{2}=0.8 ; p=0.041\right)$. No correlation was found for FBN1_004 $\left(R^{2}=0.02 ; p=0.79\right)$ (Figure 1c).

\subsection{Expression of FBN1 Isoforms in Skin Fibroblasts from MFS Patients}

In MFS skin fibroblasts, as expected, the total level of FBN1 mRNA was significantly lower than in control fibroblasts $(p<0.0001)$. The relative expression of isoform FBN1_001 was significantly reduced $(p<0.0001)$ compared to control (Figure 1b). No significant difference compared to controls was observed for the relative expressions of FBN1_004 ( $p=0.064)$ and FBN1_009 $(p=0.27)$ (Figure 2a).

(a)

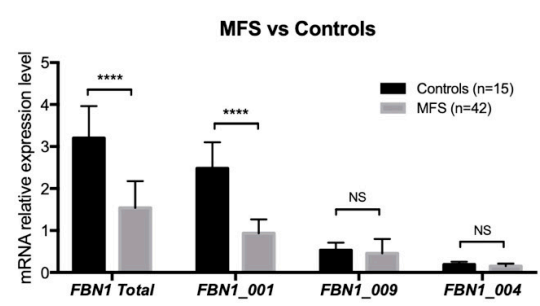

(b)

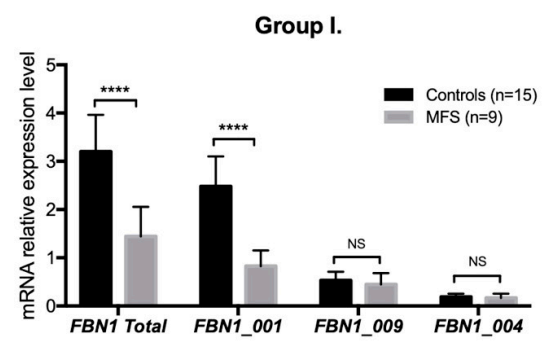

(c)

Group II.

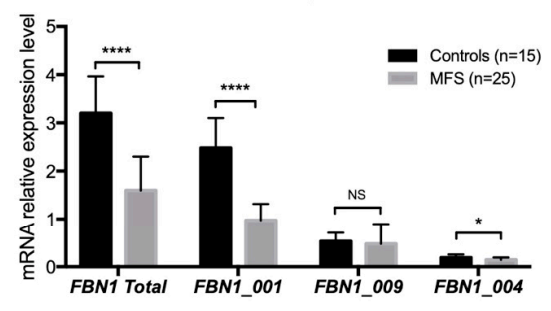

(d)

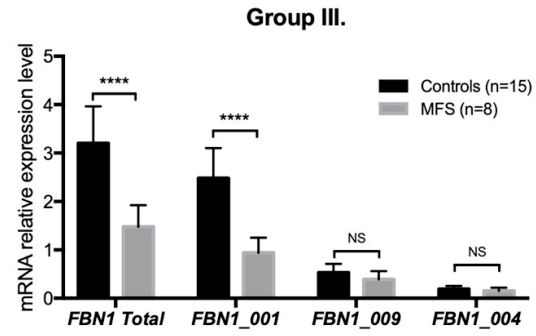

Figure 2. $F B N 1$ isoform expressions and $F B N 1$ mutation locations. (a) $F B N 1$ isoform expression levels in controls and MFS patients. A significant decrease in expression was found for MFS patients regarding FBN1 total expression $(p<0.0001)$ and FBN1_001 expression $(p<0.0001)$. No significant difference was found for the other two isoforms (FBN1_004 and FBN1_009). (b) Isoform expression levels in group I. A significant decrease in expression was found for $F B N 1$ total expression $(p<0.0001)$ and FBN1_001 expression $(p<0.0001)$. No difference was observed for FBN1_004 and FBN1_009. (c) Isoform expression levels in group II. A significant decrease in expression was found for $F B N 1$ total expression $(p<0.0001)$, FBN1_001 expression $(p<0.0001)$, and FBN1_004 expression $(p=0.015)$. No difference was observed for FBN1_009. (d) Isoform expression levels in group III. A significant decrease in expression was found for FBN1 total expression $(p<0.0001)$ and FBN1_001 expression $(p<0.0001)$. No difference was observed for FBN1_004 and FBN1_009. NS: $p>0.05 ; *$ : $p<0.05 ;{ }^{* * * *}: p<0.0001$. 


\subsection{FBN1 Isoform Expressions and FBN1 Mutation Locations}

The possible impact on FBN1 isoform expression of the location of the mutation in the FBN1 gene was tested. MFS patients were divided into three groups: group I (mutations between exons 1 and 37, included), group II (exons 38 to 63, included), and group III (exons 64 to 66, included) (Figure 1a). For all groups compared to controls, a significant decrease in total FBN1 expression and isoform FBN1_001 expression was observed $(p<0.0001)$ (Figure 2a-d). FBN1_004 expression was reduced in group II ( $p=0.03)$, while no difference in expression was observed in group III (Figure 2c). No difference in expression was observed for isoform FBN1_009 in all three groups (Figure 2a-d).

\subsection{Correlation between FBN1 Isoform Expressions in Skin Fibroblasts from Controls and MFS Patients}

The possible existence of a correlation between FBN1 isoform expressions was tested both in controls and in MFS patients. In controls, a positive correlation was only observed between the expressions of isoforms FBN1_001 and FBN1_009 $\left(R^{2}=0.39, p=0.012\right)$ (Figure 3a). Conversely, all isoform expressions were correlated in MFS patients; a positive correlation was found between the expression of isoforms FBN1_001 and FBN1_009 $\left(R^{2}=0.32, p<0.0001\right)$, between the expression of isoforms FBN1_001 and FBN1_004 $\left(R^{2}=0.43, p<0.0001\right)$, and between the expression of isoforms FBN1_004 and FBN1_009 $\left(R^{2}=0.13, p=0.012\right)$ (Figure 3b).

(a) Controls
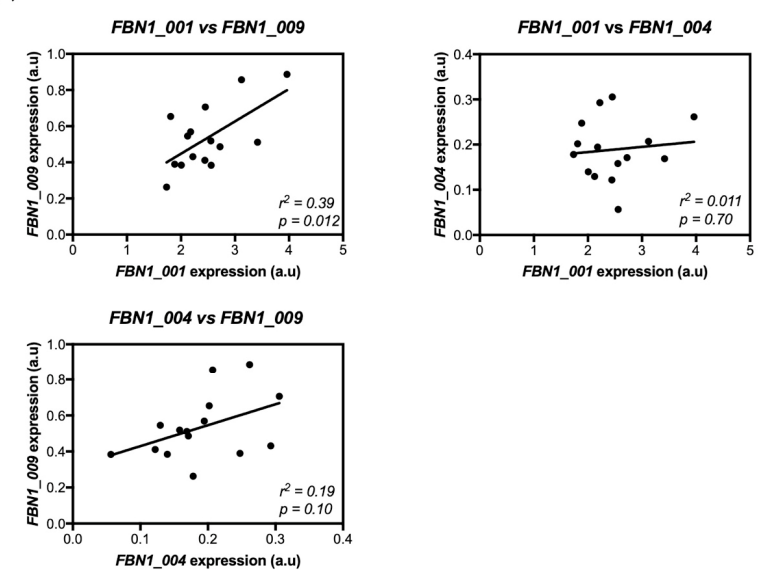

(b) MFS patients
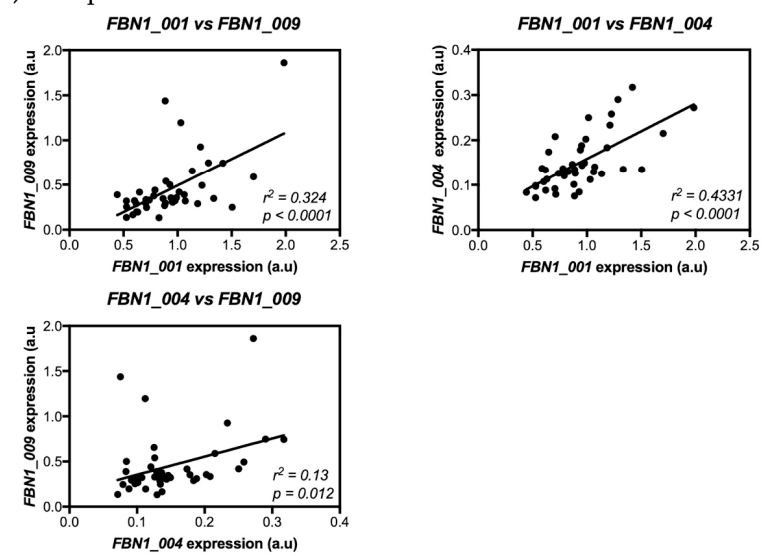

Figure 3. Correlation of expression levels between FBN1 isoforms. (a) Expression levels were assessed in controls. A positive correlation was found between FBN1_001 and FBN1_009 expressions. No correlations were found between FBN1_004 expression and that of the other two isoforms (FBN1_001 and FBN1_009). (b) Expression levels were assessed in MFS patients. A positive correlation was found between the three isoforms. 


\subsection{Relationship between FBN1 Isoform Expression and the Clinical Severity of MFS Features}

Significantly lower levels of FBN1_004 $(p=0.0221)$ and FBN1_009 $(p=0.0071)$ expression were found in patients with ectopia lentis as compared to patients without ectopia lentis (Figure 4). No correlation was found between total FBN1 or FBN1_001 expression with ectopia lentis. A correlation was also found between FBN1_009 expression and myopia. Patients with myopia had a higher FBN1_009 expression compared to patients without myopia $(p=0.03)$. No other relationship was found between FBN1 isoform expression levels and all other features of the syndrome, notably the cardiovascular, skeletal, and pulmonary systems.

(a)

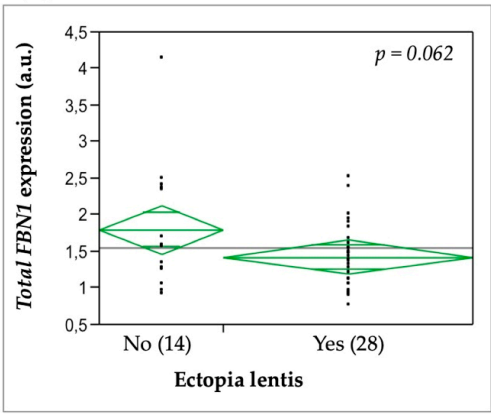

(c)

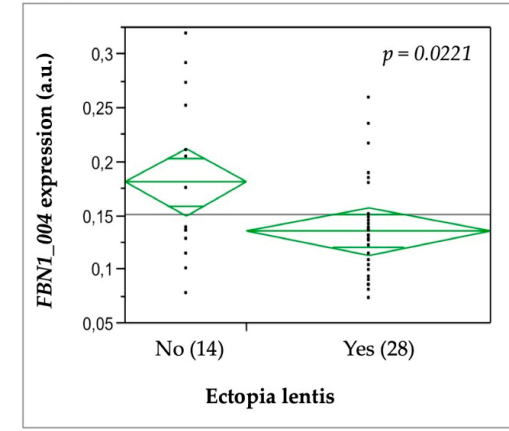

(b)

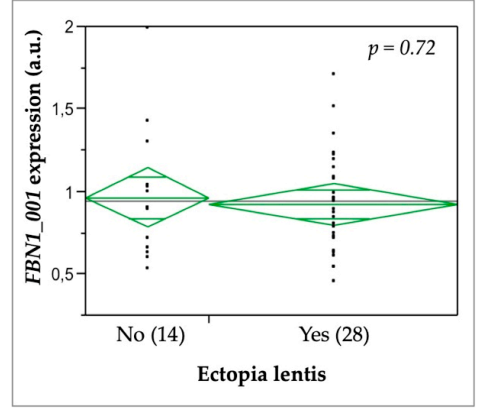

(d)

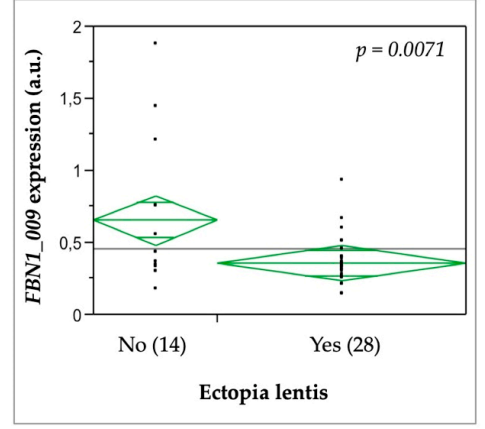

Figure 4. FBN1 mRNA expression levels in MFS patients with or without ectopia lentis. (a) Total FBN1 expression; (b) FBN1_001 expression; (c) FBN1_004 expression; (d) FBN1_009 expression. Diamond plots display the means of each group. The line across each diamond represents the group mean, and the vertical span of each diamond represents the $95 \%$ confidence interval for each group.

\section{Discussion}

This study investigated, for the first time, the expression profile of $F B N 1$ isoforms not only in a large number of control subjects, but also in skin and adventitial fibroblast pairs from patients. Previously, Burchett et al. (2011) studied FBN1 isoform expression profiles in human brain and skeletal muscle, as well as total RNA from human fetal skeletal muscle, brain, liver, aorta, lung, skin, and heart. They detected two splice variants resulting from the inclusion of cryptic exons in intron 54 or 57 , but only the latter isoform was further studied. Although this isoform is no longer referenced in the Ensembl database, it could possibly contain the cryptic exon found in FBN1_004. However, Burchett et al. (2011) did not identify the $5^{\prime}$ truncation of exons 1 to 37 that leads to isoform FBN1_004. The results of our study show that, in skin and adventitial fibroblasts, only three of the eight isoforms referenced in databases exist: FBN1_001, FBN1_004, and FBN1_009. The main isoform is FBN1_001 and corresponds to the reference transcript. The other two isoforms are shorter. The FBN1_004 isoform comprises 30 exons (with a specific exon 21), which match the last 29 exons of isoform FBN1_001. As per the Ensembl database, no information for its promoter and regulation regions, as well as its start codon, is available, since the $5^{\prime}$ untranslated region (UTR) sequence of the 
isoform is incomplete. Therefore, the precise reading frame of FBN1_004 isoform is unknown. Isoform FBN1_009 comprises four exons with a specific exon 1, and the last three exons of the FBN1 gene. It is referenced in Ensembl as a processed and non-coding transcript. Its function remains unknown. In our control cell models (adventitial and skin fibroblasts), FBN1_009 expression was higher than that of FBN1_004, contrary to what is reported in the GTEx database. Furthermore, we observed a significant correlation only between the expressions of the two isoforms FBN1_001 and FBN1_009. A single promoter region is described for the FBN1 gene and its isoforms. This region contains three distinct exons (exons A, B, and C) that are alternatively spliced with the first coding exon of the gene [17]. The three exons are embedded in an approximately 1.8-kb cytosine-phosphate-guanine (CpG) island that contains multiple putative specificity protein 1 (Sp-1) binding sites [18,19]. The promoter that includes exon A appears to be the most commonly used [18-21] and this may partly explain the positive expression correlation between the two isoforms FBN1_001 and FBN1_009. In the same way, the lack of expression correlation between FBN1_004 and the other isoforms could result from an alternative splicing mechanism via another promoter exon (B or $\mathrm{C}$ ).

As previously described [12], total FBN1 expression was significantly reduced in MFS patients as expected in PTC-mutation carriers. This decrease was only due to a significant decrease in FBN1_001 expression, while no significant difference was observed for FBN1_004 and FBN1_009 expressions. The effect on FBN1_001 expression was expected since it is the main isoform. Conversely, since these results combine the overall effect of mutations distributed throughout the gene, it is possible that mutations affecting specific exons lead to different isoform expression patterns, notably for isoforms FBN1_004 and FBN1_009. To test this hypothesis, MFS patients were divided into three groups based on the location of the mutation: mutations in the $3^{\prime}$ region common to all three isoforms, mutations between exons 38 and 63 (region common to isoforms FBN1_001 and FBN1_004), and mutations in the $5^{\prime}$ region from exon 1 to 37 (isoform FBN1_001 exon-specific region). Expression of this last isoform was significantly reduced in all mutation groups. Furthermore, FBN1_004 expression levels were lower in MFS patients carrying a mutation between exons 38 and 63 (group II) compared to the two other groups of patients, while FBN1_009 expression showed no difference between mutation groups. These two results could be explained by the presence or absence of nonsense-mediated mRNA decay (NMD) depending on the isoform. NMD is the main biological process for RNA degradation that targets only actively translated mRNAs that carry a PTC [22]. Therefore, this process will not affect isoform FBN1_009 since it is not translated. However, it should affect isoforms FBN1_001 and FBN1_004. Therefore, NMD could explain the decrease observed in the expression of both these isoforms in MFS patients. Interestingly, the expression of these isoforms is correlated in patients. This could reflect the existence of a specific mechanism that is activated to compensate for the impact of the mutation in the gene.

As previously shown, FBN1 expression level is a good surrogate endpoint for clinical severity, as lower FBN1 expression levels are associated with ectopia lentis and pectus excavatum [12]. In our study, we found a trend toward a correlation between total FBN1 expression and the presence of ectopia lentis. This could be explained by different sample sizes, since we could only test a subset of the original sample in this study and we have, therefore, a lower statistical power. Furthermore, the original study design did not take into account the existence of the three isoforms. Indeed, in Aubart et al. (2015), FBN1 expression was quantified as a mean of the whole FBN1 expression evaluated with two sets of primers located in exons 2 and 47. Therefore, correlation analyses were retrospectively performed, firstly, only using expression data obtained with exon 2 primers and, secondly, only using the data from exon 47 primers. This second analysis revealed a correlation with ectopia lentis in agreement with the original results, while no correlation was found with data from exon 2 primers. Therefore, our present results would indicate that the correlation observed with ectopia lentis is supported by isoform FBN1_004. Furthermore, a significant association was found in patients between the expressions of FBN1_004 and FBN1_009. This suggests that variations in the expression of both these isoforms have an impact on the pathogenesis of ectopia lentis. 


\section{Conclusions}

In conclusion, in our study, we showed, for the first time, that FBN1 alternative splicing could be a mechanism underlying MFS clinical variability. Further data must now be accrued to determine the sequences involved in the overall regulation of the expression of the FBN1 gene and its three alternative transcripts.

Author Contributions: Conceptualization, M.A., G.J., and C.B.; methodology, L.B., M.-S.G., N.H., and P.A.; formal and statistical analysis, G.J. and L.B.; resources, P.A., N.H., G.J., and C.B; writing-original draft preparation, L.B., C.B., and M.A.; writing—review and editing, G.J., L.B., and C.B.; funding acquisition, M.A., C.B., and G.J.

Funding: This research was funded by DHU FIRE (Emergence 2 project for M.A.), Programme Hospitalier de Recherche Clinique (AOM10108 and CRC15014 for C.B.), Agence Nationale de la Recherche (NONAGES, ANR-14-CE15-0012-01 for G.J.), Fédération de Cardiologie (for G.J.), and Société Française de Cardiologie (for G.J.).

Conflicts of Interest: The authors declare no conflict of interest. The funders had no role in the design of the study; in the collection, analyses, or interpretation of data; in the writing of the manuscript, and in the decision to publish the results.

\section{References}

1. Collod-Béroud, G.; Béroud, C.; Adès, L.; Black, C.; Boxer, M.; Brock, D.J.; Godfrey, M.; Hayward, C.; Karttunen, L.; Milewicz, D.; et al. Marfan Database (second edition): software and database for the analysis of mutations in the human FBN1 gene. Nucleic Acids Res. 1997, 25, 147-150. [CrossRef] [PubMed]

2. Loeys, B.L.; Dietz, H.C.; Braverman, A.C.; Callewaert, B.L.; De Backer, J.; Devereux, R.B.; Hilhorst-Hofstee, Y.; Jondeau, G.; Faivre, L.; Milewicz, D.M.; et al. The revised Ghent nosology for the Marfan syndrome. J. Med. Genet. 2010, 47, 476-485. [CrossRef] [PubMed]

3. Baudhuin, L.M.; Kotzer, K.E.; Lagerstedt, S.A. Increased frequency of FBN1 truncating and splicing variants in Marfan syndrome patients with aortic events. Genet. Med. 2015, 17, 177-187. [CrossRef] [PubMed]

4. Becerra-Muñoz, V.M.; Gómez-Doblas, J.J.; Porras-Martín, C.; Such-Martínez, M.; Crespo-Leiro, M.G.; Barriales-Villa, R.; de Teresa-Galván, E.; Jiménez-Navarro, M.; Cabrera-Bueno, F. The importance of genotype-phenotype correlation in the clinical management of Marfan syndrome. Orphanet J. Rare Dis. 2018, 13, 16. [CrossRef] [PubMed]

5. Faivre, L.; Collod-Beroud, G.; Loeys, B.L.; Child, A.; Binquet, C.; Gautier, E.; Callewaert, B.; Arbustini, E.; Mayer, K.; Arslan-Kirchner, M.; et al. Effect of mutation type and location on clinical outcome in 1,013 probands with Marfan syndrome or related phenotypes and FBN1 mutations: An international study. Am. J. Hum. Genet. 2007, 81, 454-466. [CrossRef] [PubMed]

6. Franken, R.; Groenink, M.; de Waard, V.; Feenstra, H.M.A.; Scholte, A.J.; van den Berg, M.P.; Pals, G.; Zwinderman, A.H.; Timmermans, J.; Mulder, B.J.M. Genotype impacts survival in Marfan syndrome. Eur. Heart J. 2016, 37, 3285-3290. [CrossRef] [PubMed]

7. Franken, R.; Teixido-Tura, G.; Brion, M.; Forteza, A.; Rodriguez-Palomares, J.; Gutierrez, L.; Garcia Dorado, D.; Pals, G.; Mulder, B.J.; Evangelista, A. Relationship between fibrillin-1 genotype and severity of cardiovascular involvement in Marfan syndrome. Heart 2017, 103, 1795-1799. [CrossRef] [PubMed]

8. Rommel, K.; Karck, M.; Haverich, A.; von Kodolitsch, Y.; Rybczynski, M.; Müller, G.; Singh, K.K.; Schmidtke, J.; Arslan-Kirchner, M. Identification of 29 novel and nine recurrent fibrillin-1 (FBN1) mutations and genotype-phenotype correlations in 76 patients with Marfan syndrome. Hum. Mutat. 2005, 26, 529-539. [CrossRef] [PubMed]

9. Schrijver, I.; Liu, W.; Odom, R.; Brenn, T.; Oefner, P.; Furthmayr, H.; Francke, U. Premature termination mutations in FBN1: Distinct effects on differential allelic expression and on protein and clinical phenotypes. Am. J. Hum. Genet. 2002, 71, 223-237. [CrossRef] [PubMed]

10. Takeda, N.; Inuzuka, R.; Maemura, S.; Morita, H.; Nawata, K.; Fujita, D.; Taniguchi, Y.; Yamauchi, H.; Yagi, H.; Kato, M.; et al. Impact of pathogenic FBN1 variant types on the progression of aortic disease in patients with Marfan syndrome. Circ. Genom. Precis. Med. 2018, 11, e002058. [CrossRef] [PubMed]

11. Aubart, M.; Gazal, S.; Arnaud, P.; Benarroch, L.; Gross, M.-S.; Buratti, J.; Boland, A.; Meyer, V.; Zouali, H.; Hanna, N.; et al. Association of modifiers and other genetic factors explain Marfan syndrome clinical variability. Eur. J. Hum. Genet. 2018. [CrossRef] [PubMed] 
12. Aubart, M.; Gross, M.-S.; Hanna, N.; Zabot, M.-T.; Sznajder, M.; Detaint, D.; Gouya, L.; Jondeau, G.; Boileau, C.; Stheneur, C. The clinical presentation of Marfan syndrome is modulated by expression of wild-type FBN1 allele. Hum. Mol. Genet. 2015, 24, 2764-2770. [CrossRef] [PubMed]

13. Burchett, M.E.; Ling, I.-F.; Estus, S. FBN1 isoform expression varies in a tissue and development-specific fashion. Biochem. Biophys. Res. Commun. 2011, 411, 323-328. [CrossRef] [PubMed]

14. Benarroch, L.; Aubart, M.; Gross, M.-S.; Jacob, M.-P.; Arnaud, P.; Hanna, N.; Jondeau, G.; Boileau, C. Marfan syndrome variability: Investigation of the roles of sarcolipin and calcium as potential transregulator of FBN1 expression. Genes 2018, 9, 421. [CrossRef] [PubMed]

15. Ensembl Genome Browser 94. Available online: https://www.ensembl.org/index.html (accessed on 7 January 2019).

16. GTEx Portal. Available online: https://gtexportal.org/home/ (accessed on 7 January 2019).

17. Corson, G.M.; Chalberg, S.C.; Dietz, H.C.; Charbonneau, N.L.; Sakai, L.Y. Fibrillin binds calcium and is coded by cDNAs that reveal a multidomain structure and alternatively spliced exons at the $5^{\prime}$ end. Genomics 1993, 17, 476-484. [CrossRef] [PubMed]

18. Guo, G.; Bauer, S.; Hecht, J.; Schulz, M.H.; Busche, A.; Robinson, P.N. A short ultraconserved sequence drives transcription from an alternate FBN1 promoter. Int. J. Biochem. Cell Biol. 2008, 40, 638-650. [CrossRef] [PubMed]

19. Guo, G.; Rödelsperger, C.; Digweed, M.; Robinson, P.N. Regulation of fibrillin-1 gene expression by Sp1. Gene 2013, 527, 448-455. [CrossRef] [PubMed]

20. Biery, N.J.; Eldadah, Z.A.; Moore, C.S.; Stetten, G.; Spencer, F.; Dietz, H.C. Revised genomic organization of FBN1 and significance for regulated gene expression. Genomics 1999, 56, 70-77. [CrossRef] [PubMed]

21. Summers, K.M.; Bokil, N.J.; Baisden, J.M.; West, M.J.; Sweet, M.J.; Raggatt, L.J.; Hume, D.A. Experimental and bioinformatic characterisation of the promoter region of the Marfan syndrome gene, FBN1. Genomics 2009, 94, 233-240. [CrossRef] [PubMed]

22. Lykke-Andersen, S.; Jensen, T.H. Nonsense-mediated mRNA decay: An intricate machinery that shapes transcriptomes. Nat. Rev. Mol. Cell Biol. 2015, 16, 665-677. [CrossRef] [PubMed]

(C) 2019 by the authors. Licensee MDPI, Basel, Switzerland. This article is an open access article distributed under the terms and conditions of the Creative Commons Attribution (CC BY) license (http:/ / creativecommons.org/licenses/by/4.0/). 